\title{
ELUSIVE CODES IN HAMMING GRAPHS
}

\author{
DANIEL R. HAWTIN ${ }^{凶}$, NEIL I. GILLESPIE and CHERYL E. PRAEGER
}

(Received 22 August 2012; accepted 5 December 2012; first published online 28 February 2013)

\begin{abstract}
We consider a code to be a subset of the vertex set of a Hamming graph. We examine elusive pairs, code-group pairs where the code is not determined by knowledge of its set of neighbours. We construct a new infinite family of elusive pairs, where the group in question acts transitively on the set of neighbours of the code. In these examples, the alphabet size always divides the length of the code. We show that there is no elusive pair for the smallest set of parameters that does not satisfy this condition. We also pose several questions regarding elusive pairs.
\end{abstract}

2010 Mathematics subject classification: primary 94B60; secondary 05E18.

Keywords and phrases: elusive codes, permutation codes, powerline communication, neighbour transitive codes, automorphism groups.

\section{Introduction and motivation}

Given a group that fixes setwise the set of neighbours of a certain code, the question of whether the group necessarily fixes the code setwise was considered by the second and third authors in [8]. In particular, they considered codes in a Hamming graph $\Gamma=H(m, q)$, in which each vertex, and in particular each codeword, is an $m$-tuple with entries from a set $Q$ of size $q$. In this context, a codeword $\alpha$ with a single symbol changed, that is, a single error introduced, corresponds to a vertex $v$ in $\Gamma$ adjacent to $\alpha$. We refer to $v$ as a neighbour of $\alpha$, and for a code $C$, the set of neighbours of $C$, denoted by $\Gamma_{1}(C)$, consists of all vertices of $\Gamma$ which are not in $C$, but are adjacent to at least one element of $C$.

The group fixing $\Gamma_{1}(C)$ setwise is a subgroup, $G$, of the automorphism group, $\operatorname{Aut}(\Gamma)$, of $\Gamma$. Whether $G$ fixes $C$ setwise depends on certain parameters of the code. One such parameter is the minimum distance, $\delta$, defined to be the smallest distance in $\Gamma$ between distinct codewords in $C$. In particular, by [8, Theorem 1], if $C$ is a code in $H(m, q)$ with $\delta \geq 3$ such that $G$ does not fix $C$ setwise, then one of the following holds:

(1) $\delta=4, q=2$ and $m$ is even;

(2) $\delta=3$, and $m(q-1)$ is even.

The second author is supported by the Australian Research Council Federation Fellowship FF0776186 of the third author.

(c) 2013 Australian Mathematical Publishing Association Inc. 0004-9727/2013 \$16.00 
The paper [8] exhibits an infinite family of codes and groups with the parameters of (1), but no examples for case (2) are given. The aim of this paper is to provide infinitely many examples for case (2). All of our examples have $m$ a multiple of $q$ and we pose several questions about the parameters and properties of such codes. We make the following definition.

Definition 1.1. Let $C$ be a code in $\Gamma=H(m, q)$ with minimum distance $\delta$, and let $X \leq \operatorname{Aut}(\Gamma)$ such that $X$ fixes $\Gamma_{1}(C)$ setwise, but does not fix $C$ setwise. Then we call $(C, X)$ an elusive pair, with parameters $(m, q, \delta)$.

The paper [8] contains no comment on elusive pairs with the parameters of (2). However, the discussion following [6, Problem 11.1] asks if there exist elusive pairs with $\delta=3$ and $m(q-1)$ even. We prove the following theorem.

Theorem 1.2. Let $q \geq 3$ and $m$ be divisible by $q$. Then there exists a code $C$ with minimum distance $\delta=3$, and a group $X$ such that $(C, X)$ is an elusive pair with parameters $(m, q, 3)$, and $X$ is transitive on $\Gamma_{1}(C)$.

We prove further in Section 3.4 that there are no elusive pairs with parameters $(4,3,3)$. Since we require $m \geq 3$ in order to have $\delta=3$, and $(3,4,3)$ does not satisfy $m(q-1)$ even, this is the smallest set of parameters satisfying condition (2), where $q$ does not divide $m$. The following question, however, remains unanswered.

Question 1.3. Do there exist elusive pairs with parameters $(m, q, 3)$ such that $m$ is not a multiple of $q$ ? More generally we ask for a determination of the possible parameters of elusive pairs.

1.1. Commentary and further questions. An assumption frequently made in coding theory is that during transmission of an encoded message, the probability of an error occurring is independent of the symbol sent and its position in the message. In [7, 8], the second and third authors introduce neighbour transitivity as a group theoretic analogue of this assumption. A code $C$ is defined to be neighbour transitive, or $X$-neighbour transitive if we wish to specify the group, if there exists a subgroup $X \leq \operatorname{Aut}(\Gamma)$ that fixes setwise and acts transitively on both $C$ and $\Gamma_{1}(C)$.

For the infinite family of examples from [8, Section 5], it is shown, in that paper, that there exists a group $X$ such that $X$ is transitive on the set of neighbours of the code. Moreover, it is contained in a larger code which shares the same neighbour set, and is, in fact, $X$-neighbour transitive. The construction we give in Section 3.1 produces an $X$-neighbour transitive code, $C^{\prime}$, with minimum distance $\delta^{\prime}=2$, such that $C^{\prime}$ contains a code, $C$, with $\delta=3$ and $\Gamma_{1}(C)=\Gamma_{1}\left(C^{\prime}\right)$. It follows that $X$ acts transitively on $\Gamma_{1}(C)$, but does not fix $C$ setwise, and thus $(C, X)$ is an elusive pair.

For each elusive pair $(C, X)$ in Sections 3.1 and 3.2, and for those constructed in [8, Section 5], we observe that if $x \in X$ does not fix $C$ setwise, then there is only one possibility for the image, $C^{x}$. Thus, under $X, C$ has two images, the original code $C$ and $C^{x}$. 
Question 1.4. Does there exist an elusive pair $(C, X)$ such that $C$ has more than two images under $X$ ? More generally, if $r=\left|\left\{C^{x} \mid x \in X\right\}\right|$, what values of $r$ are possible?

We also note that, in all examples mentioned thus far, not only is $X$ transitive on $\Gamma_{1}(C)$, but $C$ is $X_{C}$-neighbour transitive, where $X_{C}$ is the subgroup of $X$ fixing $C$ setwise. However this is not true in general. In Section 3.3 we construct a family of elusive pairs $(C, X)$, where $X_{C}$ is not transitive on $C$ and $X$ is not transitive on $\Gamma_{1}(C)$. In fact, for all of these examples $(C, X)$ there is no larger group $X^{\prime}$ such that $\left(C, X^{\prime}\right)$ is an elusive pair (see Proposition 3.10).

Another interesting feature of the elusive pairs $(C, X)$ in Sections 3.1 and 3.2, and also those in [8], is that if $x \in X$ does not fix $C$ setwise, then $C^{x}$ and $C$ are disjoint. The family of examples in Section 3.3 do not have this property. However they are not $X_{C}$-neighbour transitive.

Question 1.5. If $(C, X)$ is an elusive pair and $C$ is $X_{C}$-neighbour transitive, is it true that, for each $x \in X$, either $C^{x}=C$ or $C$ and $C^{x}$ are disjoint?

\section{Notation}

2.1. Hamming graphs. Let $C$ be a code of ordered $m$-tuples over an alphabet, $Q$, of size $q$. The Hamming graph, $\Gamma=H(m, q)$, has vertex set consisting of all $m$-tuples with entries from $Q$, with an edge existing between $m$-tuples which differ in exactly one position. The Hamming distance, $d(\alpha, \beta)$, between two vertices, $\alpha, \beta \in \Gamma$, is defined as the number of entries in which the two vertices differ. For a code $C$, the minimum distance, $\delta$, is defined as $\delta=\min \{d(\alpha, \beta) \mid \alpha, \beta \in C, \alpha \neq \beta\}$. For a vertex $\alpha \in \Gamma$, we denote the set of vertices which are at distance $r$ from $\alpha$ by $\Gamma_{r}(\alpha)=\{\beta \in \Gamma \mid d(\alpha, \beta)=r\}$. We call $\Gamma_{1}(\alpha)$ the set of neighbours of $\alpha$.

For a vertex $\alpha \in \Gamma$, define $d(\alpha, C)=\min \{d(\alpha, \beta) \mid \beta \in C\}$. This allows us to define the covering radius, $\rho=\max \{d(\alpha, C) \mid \alpha \in \Gamma\}$, and for any $r \leq \rho$ we define $\Gamma_{r}(C)=\{\alpha \in$ $\Gamma \mid d(\alpha, C)=r\}$. We refer to $\Gamma_{1}(C)$ as the set of neighbours of $C$. Note that if $\delta \geq 2$, $\Gamma_{1}(C)=\bigcup_{\alpha \in C} \Gamma_{1}(\alpha)$.

The automorphism group of the Hamming graph, Aut $(\Gamma)$, is the semi-direct product $N \rtimes L$, where $N \cong S_{q}^{m}$ and $L \cong S_{m}$, see [4, Theorem 9.2.1]. Let $g=\left(g_{1}, \ldots, g_{m}\right) \in N$, $\sigma \in L$ and $\alpha=\left(\alpha_{1}, \ldots, \alpha_{m}\right) \in \Gamma$. Then $g$ and $\sigma$ act on $\alpha$ as follows:

$$
\alpha^{g}=\left(\alpha_{1}^{g_{1}}, \ldots, \alpha_{m}^{g_{m}}\right) \quad \text { and } \quad \alpha^{\sigma}=\left(\alpha_{1^{\sigma^{-1}}}, \ldots, \alpha_{m^{\sigma^{-1}}}\right) .
$$

The automorphism group of a code $C \subseteq \Gamma$ is defined to be the setwise stabiliser of $C$ in $\operatorname{Aut}(\Gamma)$, and denoted by $\operatorname{Aut}(C)$.

2.2. Permutation codes. Let $Q=\{1, \ldots, q\}$ and $S_{q}$ be the symmetric group of $Q$. For any permutation $g \in S_{q}$, we associate with it the vertex $\alpha(g)=\left(1^{g}, \ldots, q^{g}\right)$ in $H(q, q)$. Furthermore, for $T \subseteq S_{q}$, we define the permutation code $C(T)$ to be $C(T)=$ $\{\alpha(g) \mid g \in T\}$.

Permutation codes were first studied in the 1970s, in particular by Blake et al. [3], but have recently gained attention due to a potential application in powerline 
communication, where information is transmitted as a string of frequencies through existing electrical infrastructure. This approach presents extra problems to consider. For instance, one needs the power output to remain as constant as possible, as well as there being extra noise considerations to take into account. Permutation codes have been suggested as a solution to both of these problems, see [5, 11]. For an overview of the subject see [9]. Bailey gives a decoding algorithm for permutation codes generated by groups in [1].

In [2], Blake shows how to find the minimum distance of any permutation code constructed from a sharply $k$-transitive group. A group $G$ acting on a set $\Omega$ is sharply $k$ transitive if for any two ordered $k$-tuples of distinct points, there is a unique element of $G$ mapping the first to the second. So the identity element is the unique element fixing $k$ points, and thus, for $g_{1}, g_{2} \in G$ with $g_{1} \neq g_{2}$, we have $d\left(\alpha\left(g_{1}\right), \alpha\left(g_{2}\right)\right) \geq q-k+1$. So $\delta \geq q-k+1$. For example, $S_{q}$ is sharply $(q-1)$-transitive, and if we let $g=(12) \in S_{q}$ then $d(\alpha(1), \alpha(g))=2$, thus $C\left(S_{q}\right)$ has minimum distance 2. Also $A_{q}$ is sharply $(q-2)$ transitive, and letting $g=(123) \in A_{q}$ we have $d(\alpha(1), \alpha(g))=3$, so $C\left(A_{q}\right)$ has minimum distance 3 . In the same paper, Blake also briefly outlines a decoding algorithm for $C\left(A_{q}\right)$.

Let $i, j \in Q, i \neq j$ and $g \in S_{q}$, and define $v(\alpha(g), i, j)$ to be the vertex in $H(q, q)$ with $k$ th entry given by

$$
\left.v(\alpha(g), i, j)\right|_{k}= \begin{cases}k^{g} & \text { if } k \neq i, \\ j^{g} & \text { if } k=i .\end{cases}
$$

If $i \neq j$, then $v(\alpha(g), i, j)$ differs from $\alpha(g)$ at the $i$ th entry only, and thus $v(\alpha(g), i, j) \in$ $\Gamma_{1}(\alpha(g))$. Each of the $q(q-1)$ neighbours of $\alpha(g)$ is of this form; there are $q$ choices for $i$ and, given $i$, there are $q-1$ choices for $j^{g} \neq i^{g}$, and hence of $j$.

For $y \in S_{q}$, we denote $x_{y}=(y, \ldots, y) \in N$, and define $\operatorname{Diag}_{q}\left(S_{q}\right)=\left\{x_{y} \mid y \in S_{q}\right\} \leq N$. Also, for $z \in S_{q}$ let $\sigma(z)$ be the permutation in the top group $L$, induced by $z$. Let $g, y, z \in S_{q}, i \neq j, x_{y}=(y, \ldots, y) \in \operatorname{Diag}_{q}\left(S_{q}\right)$ and $\sigma(z) \in L$. Then, by [6, Lemmas 5.1.1 and 5.1.1.3],

$$
\alpha(g)^{x_{y} \sigma(z)}=\alpha\left(z^{-1} g y\right) \quad \text { and } \quad v(\alpha(g), i, j)^{x_{y} \sigma(z)}=v\left(\alpha\left(z^{-1} g y\right), i^{z}, j^{z}\right) .
$$

\section{Elusive pairs}

In this section we construct the examples that contribute to the proof of Theorem 1.2, as well as showing that there is no elusive pair for the smallest set of parameters where $q$ does not divide $m$.

3.1. Example 1. We show that $\left(C\left(A_{q}\right), \operatorname{Diag}_{q}\left(S_{q}\right) \rtimes L\right)$ is an elusive pair, with parameters $(q, q, 3)$. We begin by showing that the larger code, $C\left(S_{q}\right)$, with minimum distance two, has the same neighbour set as $C\left(A_{q}\right)$.

Lemma 3.1. For distinct $i, j \in\{1, \ldots, q\}$ and $g \in A_{q}$, let $g^{\prime}=(i j) g$. Then $g^{\prime} \in S_{q} \backslash A_{q}$ and $v(\alpha(g), i, j)=v\left(\alpha\left(g^{\prime}\right), j, i\right)$. Thus $\Gamma_{1}\left(C\left(A_{q}\right)\right)=\Gamma_{1}\left(C\left(S_{q} \backslash A_{q}\right)\right)=\Gamma_{1}\left(C\left(S_{q}\right)\right)$. 
Proof. Clearly $g^{\prime} \in S_{q} \backslash A_{q}$. We have

$$
\begin{aligned}
\left.v(\alpha(g), i, j)\right|_{k} & = \begin{cases}k^{g} & \text { if } k \neq i, \\
j^{g} & \text { if } k=i\end{cases} \\
& = \begin{cases}k^{g^{\prime}} & \text { if } k \neq i, j, \\
i^{g^{\prime}} & \text { if } k=i, \\
i^{g^{\prime}} & \text { if } k=j\end{cases} \\
& = \begin{cases}k^{g^{\prime}} & \text { if } k \neq j, \\
i^{g^{\prime}} & \text { if } k=j\end{cases} \\
& =\left.v\left(\alpha\left(g^{\prime}\right), j, i\right)\right|_{k} .
\end{aligned}
$$

Thus $v(\alpha(g), i, j)=v\left(\alpha\left(g^{\prime}\right), j, i\right)$, and the rest follows since $C\left(S_{q}\right)$ has minimum distance $\delta=2$.

Lemma 3.2 is a consequence of [6, Lemma 5.1.1.5], but we give a short proof here for completeness.

Lemma 3.2. The code $C\left(S_{q}\right)$ is $\left(\operatorname{Diag}_{q}\left(S_{q}\right) \rtimes L\right)$-neighbour transitive.

Proof. By (2.1), $C\left(S_{q}\right)$ is fixed setwise by $X=\operatorname{Diag}_{q}\left(S_{q}\right) \rtimes L$. Let $g_{1}, g_{2} \in S_{q}$, and let $y=g_{1}^{-1} g_{2}$. Then $\alpha\left(g_{1}\right)^{x_{y}}=\alpha\left(g_{1} g_{1}^{-1} g_{2}\right)=\alpha\left(g_{2}\right)$, by (2.1), and it follows that $X$ is transitive on $C\left(S_{q}\right)$. Now let $i_{1} \neq j_{1}, i_{2} \neq j_{2}$. Since $S_{q}$ acts 2-transitively on $Q$, there exists $z \in S_{q}$ such that $i_{1}^{z}=i_{2}$ and $j_{1}^{z}=j_{2}$. Let $y=g_{1}^{-1} z g_{2}$. Then, again using (2.1), $v\left(\alpha\left(g_{1}\right), i_{1}, j_{1}\right)^{x_{y} \sigma(z)}=v\left(\alpha\left(z^{-1} g_{1} g_{1}^{-1} z g_{2}\right), i_{1}^{z}, j_{1}^{z}\right)=v\left(\alpha\left(g_{2}\right), i_{2}, j_{2}\right)$. Therefore, $X$ acts transitively on $\Gamma_{1}\left(C\left(S_{q}\right)\right)$ and so $C\left(S_{q}\right)$ is $X$-neighbour transitive.

Lemma 3.3. Let $x=x_{y} \sigma(z) \in \operatorname{Diag}_{q}\left(S_{q}\right) \rtimes L$. Then $C\left(A_{q}\right)^{x}=C\left(z^{-1} y A_{q}\right)$. In particular, $C\left(A_{q}\right)^{x}=C\left(A_{q}\right)$ if and only if $z^{-1} y \in A_{q}$.

Proof. By (2.1), $\alpha(g)^{x}=\alpha\left(z^{-1} g y\right)=\alpha\left(z^{-1} y y^{-1} g y\right)$ for all $g \in A_{q}$. As $A_{q}$ is a normal subgroup of $S_{q}$, the assertion follows.

Corollary 3.4. The pair $\left(C\left(A_{q}\right), \operatorname{Diag}_{q}\left(S_{q}\right) \rtimes L\right)$ is an elusive pair with parameters $(q, q, 3)$.

Proof. By Lemma 3.2, $X=\operatorname{Diag}_{q}\left(S_{q}\right) \rtimes L$ is transitive on $\Gamma_{1}\left(C\left(S_{q}\right)\right)$ and so, by Lemma 3.1, $X$ is transitive on $\Gamma_{1}\left(C\left(A_{q}\right)\right)$. By Lemma 3.3, $C\left(A_{q}\right)$ is not fixed by $X$. Thus $\left(C\left(A_{q}\right), X\right)$ is an elusive pair.

For $C\left(A_{q}\right), m=q$, so $m(q-1)$ is even, and also $\delta=3$, as mentioned in Section 2.2. So $C\left(A_{q}\right)$ indeed satisfies (2). By Lemma 3.3, each element of $\operatorname{Diag}_{q}\left(S_{q}\right) \rtimes L$ either fixes both $C\left(A_{q}\right)$ and $C\left(S_{q} \backslash A_{q}\right)$ setwise, or swaps them. Note furthermore that $C\left(A_{q}\right) \cup C\left(S_{q} \backslash A_{q}\right)=C\left(S_{q}\right)$.

3.2. Example 2. The product construction of a code $C$ in $H(m, q)$ is defined in [6, Section 4.7] as follows:

$$
\operatorname{Prod}(C, l)=\left\{\left(\alpha_{1}, \ldots, \alpha_{l}\right) \mid \alpha_{i} \in C, \forall i\right\},
$$


which is a code in $H(l m, q)$. We use this construction for the next family of examples. First we set up the required notation.

Previously we used a subscript to refer simply to the $k$ th entry of a vertex; however, there is now some ambiguity. We may wish to refer to the $k$ th entry of a vertex in $H(l m, q)$, or the $k$ th entry, $\alpha_{k}$, of the $l$-tuple $\left(\alpha_{1}, \ldots, \alpha_{l}\right) \in H(l m, q)$, which is itself a vertex in $H(m, q)$. In this section we always mean the $k$ th entry in $\left(\alpha_{1}, \ldots, \alpha_{l}\right)$, so that ' $k$ th entries' are vertices of $H(m, q)$.

Let $C \subseteq H(m, q)$. By [6, Lemma 4.7.1], $C$ and $\operatorname{Prod}(C, l)$ have the same minimum distance, $\delta$ say. If $\delta \geq 2$ then, given $\alpha=\left(\alpha_{1}, \ldots, \alpha_{l}\right) \in \operatorname{Prod}(C, l)$, replacing a single $\alpha_{i}$ with $v \in \Gamma_{1}\left(\alpha_{i}\right)$ yields a neighbour of $\alpha$, which we denote by $\mu(\alpha, v, i)$, where

$$
\left.\mu(\alpha, v, i)\right|_{k}= \begin{cases}\alpha_{k} & \text { if } k \neq i \\ v & \text { if } k=i .\end{cases}
$$

There are $m(q-1)$ choices for $v$, and $l$ choices for $i$, and so all the $\operatorname{lm}(q-1)$ neighbours of $\alpha$ have this form. Given an action of $X$ on $\Gamma=H(m, q)$, we can define an action of $X \imath S_{l}$ on the cartesian product of $l$ copies of $\Gamma$. Let $\alpha=\left(\alpha_{1}, \ldots, \alpha_{l}\right)$, with each $\alpha_{i} \in \Gamma$, $\left(x_{1}, \ldots, x_{l}\right) \in X^{l}, \sigma \in S_{l}$. Then

$$
\boldsymbol{\alpha}^{\left(x_{1}, \ldots, x_{l}\right)}=\left(\alpha_{1}^{x_{1}}, \ldots, \alpha_{l}^{x_{l}}\right) \quad \text { and } \quad \boldsymbol{\alpha}^{\sigma}=\left(\alpha_{1^{\sigma^{-1}}}, \ldots, \alpha_{l^{\sigma^{-1}}}\right),
$$

and these elements act on the neighbours of $\operatorname{Prod}(C, l)$ as follows:

$$
\begin{aligned}
\left.\mu(\alpha, v, i)^{\left(x_{1}, \ldots, x_{l}\right)}\right|_{k} & = \begin{cases}\alpha_{k}^{x_{k}} & \text { if } k \neq i, \\
v^{x_{i}} & \text { if } k=i\end{cases} \\
& =\left.\mu\left(\boldsymbol{\alpha}^{\left(x_{1}, \ldots, x_{l}\right)}, v^{x_{i}}, i\right)\right|_{k} .
\end{aligned}
$$

Suppose $k^{\sigma}=n$. Then

$$
\begin{aligned}
\left.\mu(\boldsymbol{\alpha}, v, i)^{\sigma}\right|_{n}=\left.\mu(\boldsymbol{\alpha}, v, i)\right|_{k} & = \begin{cases}\alpha_{k} & \text { if } k \neq i, \\
v & \text { if } k=i\end{cases} \\
& = \begin{cases}\alpha_{n^{\sigma^{-1}}} & \text { if } n^{\sigma^{-1}} \neq i, \\
v & \text { if } n^{\sigma^{-1}}=i\end{cases} \\
& = \begin{cases}\alpha_{n^{\sigma^{-1}}} & \text { if } n \neq i^{\sigma}, \\
v & \text { if } n=i^{\sigma}\end{cases} \\
& =\left.\mu\left(\boldsymbol{\alpha}^{\sigma}, v, i^{\sigma}\right)\right|_{n},
\end{aligned}
$$

which gives

$$
\mu(\boldsymbol{\alpha}, v, i)^{\left(x_{1}, \ldots, x_{l}\right)}=\mu\left(\boldsymbol{\alpha}^{\left(x_{1}, \ldots, x_{l}\right)}, v^{x_{i}}, i\right) \quad \text { and } \quad \mu(\boldsymbol{\alpha}, v, i)^{\sigma}=\mu\left(\boldsymbol{\alpha}^{\sigma}, v, i^{\sigma}\right) .
$$

If $C$ has minimum distance $\delta \geq 3$ in $H(m, q)$, then each neighbour of $\operatorname{Prod}(C, \ell)$ has a unique representation of the form $\mu(\alpha, v, i)$. This, however, is not the case when $\delta \leq 2$. Let $C$ be a code with $\delta=2$ and $\alpha, \beta \in C$ such that $d(\alpha, \beta)=2$, and consider $v \in \Gamma_{1}(\alpha) \cap \Gamma_{1}(\beta)$. Then, for $\alpha=(\alpha, \ldots, \alpha)$ and $\beta=(\beta, \alpha, \ldots, \alpha)$ in $\operatorname{Prod}(C, l)$, it follows that $\mu(\alpha, v, 1)=\mu(\beta, v, 1)$. Gillespie [6, Lemma 4.7.3] proved the next result for codes with $\delta \geq 3$; however it is in fact true for arbitrary minimum distance. 
Lemma 3.5. Let $C$ be an $X$-neighbour transitive code in $H(m, q)$. Then $\operatorname{Prod}(C, l)$ is $X>S_{l}$-neighbour transitive in $H(l m, q)$.

Proof. It follows from (3.1) that $X^{l}$ is transitive on $\operatorname{Prod}(C, l)$ since $X$ is transitive on $C$. To map the neighbour $\mu(\alpha, v, i)$ to the neighbour $\mu\left(\beta, v^{\prime}, j\right)$, we first apply $\sigma=(i j) \in S_{l}$, so $\mu(\alpha, v, i)^{\sigma}=\mu\left(\alpha^{\sigma}, v, j\right)$. As $C$ and $\Gamma_{1}(C)$ are both $X$-orbits in $H(m, q)$, there exists $x_{k} \in X$ such that $\alpha_{k}^{x_{k}}=\beta_{k}$ for $k \neq i, j$, there exists $x_{i} \in X$ such that $\alpha_{j}^{x_{i}}=\beta_{i}$, and there exists $x_{j} \in X$ such that $v^{x_{j}}=v^{\prime}$. By letting $x=\left(x_{1}, \ldots, x_{l}\right) \in X^{l}$, it follows that $\mu(\alpha, v, i)^{\sigma x}=\mu\left(\beta, v^{\prime}, j\right)$.

The next result follows directly from Lemmas 3.2 and 3.5.

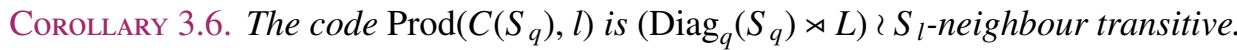

Definition 3.7. Let $C(q, l)$ be the subset of $\operatorname{Prod}\left(C\left(S_{q}\right), l\right)$ where $\left(\alpha\left(g_{1}\right), \ldots, \alpha\left(g_{l}\right)\right) \in$ $C(q, l)$ if and only if $\left|\left\{i \mid g_{i} \in A_{q}\right\}\right|$ is even.

In the remainder of this section we show that $\left(C(q, l),\left(\operatorname{Diag}_{q}\left(S_{q}\right) \rtimes L\right) \prec S_{l}\right)$ is an elusive pair.

Lemma 3.8. We have $\Gamma_{1}\left(\operatorname{Prod}\left(C\left(S_{q}\right), l\right)\right)=\Gamma_{1}(C(q, l))$, with $C(q, l)$ as in Definition 3.7.

Proof. Set $\mathcal{P}=\operatorname{Prod}\left(C\left(S_{q}\right), l\right)$ and $C=C(q, l)$. Let $\alpha=\left(\alpha\left(g_{1}\right), \ldots, \alpha\left(g_{l}\right)\right) \in \mathcal{P}, v=$ $v\left(\alpha\left(g_{k}\right), i, j\right)$ for some $i \neq j \leq q$, and $\mu=\mu(\alpha, v, k) \in \Gamma_{1}(\mathcal{P})$. Suppose $\alpha \notin C$, and let $g_{n}^{\prime}=g_{n}$ for $n \neq k$ and $g_{k}^{\prime}=(i j) g_{k}$. Since $g_{k}$ and $g_{k}^{\prime}$ have different parities, it follows that $\boldsymbol{\alpha}^{\prime}=\left(\alpha\left(g_{1}^{\prime}\right), \ldots, \alpha\left(g_{l}^{\prime}\right)\right) \in C$. Consider $\nu^{\prime}=\nu\left(\alpha\left(g_{k}^{\prime}\right), j, i\right)$ and $\boldsymbol{\mu}^{\prime}=\mu\left(\boldsymbol{\alpha}^{\prime}, \nu^{\prime}, k\right)$. By Lemma 3.1, $v(\alpha(g), i, j)=v\left(\alpha\left(g^{\prime}\right), j, i\right) \in H(q, q)$. Thus, $\boldsymbol{\mu}=\boldsymbol{\mu}^{\prime} \in \Gamma_{1}(C)$, so $\Gamma_{1}(\mathcal{P}) \subseteq$ $\Gamma_{1}(C)$. The fact that $\Gamma_{1}(C) \subseteq \Gamma_{1}(\mathcal{P})$ holds because $\mathcal{C} \subseteq \mathcal{P}$ and $\mathcal{P}$ has minimum distance 2.

Lemma 3.9. The pair $\left(C(q, l),\left(\operatorname{Diag}_{q}\left(S_{q}\right) \rtimes L\right) \imath S_{l}\right)$ is an elusive pair, with parameters $(l q, q, 3)$.

Proof. By Corollary 3.6, $X=\left(\operatorname{Diag}_{q}\left(S_{q}\right) \rtimes L\right) \imath S_{l}$ is transitive on $\Gamma_{1}\left(\operatorname{Prod}\left(C\left(S_{q}\right), l\right)\right)$, and this set is equal to $\Gamma_{1}(C(q, l))$, by Lemma 3.8. We now show that $C(q, l)$ is not fixed by $X$. Consider $\alpha=(\alpha(1), \ldots, \alpha(1)) \in C(q, l)$ and the element $x=$ $\left(x_{y}, 1, \ldots, 1\right)$ in the base group of $\left(\operatorname{Diag}_{q}\left(S_{q}\right) \rtimes L\right) \prec S_{l}$, where $y=(12) \in S_{q}$. Then $\boldsymbol{\alpha}^{x}=(\alpha((12)), \alpha(1), \ldots, \alpha(1)) \notin C(q, l)$. Thus $(C(q, l), X)$ is an elusive pair.

It remains to show that $C(q, l)$ has minimum distance 3. Let $\alpha=\left(\alpha\left(g_{1}\right), \ldots, \alpha\left(g_{l}\right)\right)$ and $\boldsymbol{\beta}=\left(\alpha\left(g_{1}^{\prime}\right), \ldots, \alpha\left(g_{l}^{\prime}\right)\right) \in C(q, l)$, with $\boldsymbol{\alpha} \neq \boldsymbol{\beta}$. If there exists $i \neq j$ such that $g_{i} \neq g_{i}^{\prime}$ and $g_{j} \neq g_{j}^{\prime}$, then $d(\boldsymbol{\alpha}, \boldsymbol{\beta}) \geq 2 \delta_{C\left(S_{q}\right)}=4$. So suppose there exists $i$ such that $g_{k}=g_{k}^{\prime}$ for all $k \neq i$. Then $g_{i}$ and $g_{i}^{\prime}$ have the same parity, and so are either both from $C\left(A_{q}\right)$ or both from $C\left(S_{q} \backslash A_{q}\right)$. Hence $d(\boldsymbol{\alpha}, \boldsymbol{\beta}) \geq \delta_{C\left(A_{q}\right)}=3$. For equality set $g_{i}=1$ and $g_{i}^{\prime}=(123)$.

This completes the proof of Theorem 1.2, as $\left(C(q, l),\left(\operatorname{Diag}_{q}\left(S_{q}\right) \rtimes L\right) \succ S_{l}\right)$ has parameters $(l q, q, 3)$. Note that each element of $\left(\operatorname{Diag}_{q}\left(S_{q}\right) \rtimes L\right) \curlywedge S_{l}$ either fixes $C(q, l)$ setwise, or sends it to the code $C^{\prime}(q, l) \subseteq \operatorname{Prod}\left(C\left(S_{q}\right), l\right)$, 
where $\alpha=\left(\alpha\left(g_{1}\right), \ldots, \alpha\left(g_{l}\right)\right) \in C^{\prime}(q, l)$ if and only if $\left|\left\{i \mid g_{i} \in A_{q}\right\}\right|$ is odd. Observe also that $C(q, l) \cup C^{\prime}(q, l)=\operatorname{Prod}\left(C\left(S_{q}\right), l\right)$.

3.3. Example 3. For $a \in Q$ we define $\beta(a)=(a, \ldots, a) \in H(m, q)$. We define the repetition code in $H(m, q)$ as

$$
\operatorname{Rep}(m, q)=\{\beta(a) \mid a \in Q\}
$$

By [7, Theorem 3.2], $\operatorname{Rep}(m, q)$ is $\operatorname{Diag}_{m}\left(S_{q}\right) \rtimes L$-neighbour transitive with minimum distance $m$. We now construct our final example, which does not share some of the properties of previous examples.

Proposition 3.10. Let $C=C\left(A_{q}\right) \cup \operatorname{Rep}(q, q), X=\operatorname{Diag}_{q}\left(S_{q}\right) \rtimes L$ and $q \geq 5$. Then $(C, X)$ is an elusive pair, $X$ is not transitive on $\Gamma_{1}(C)$ and $X$ is the setwise stabiliser in $\operatorname{Aut}(\Gamma)$ of $\Gamma_{1}(C)$.

Proof. Let $R=\operatorname{Rep}(q, q)$. Note that $\delta_{C\left(A_{q}\right)}=3$ and $\delta_{R}=q$. If $\alpha \in C\left(A_{q}\right)$ and $\beta \in R$ then $d(\alpha, \beta)=q-1 \geq 4$, so $\Gamma_{1}(C)=\Gamma_{1}\left(C\left(A_{q}\right)\right) \cup \Gamma_{1}(R)$. By Lemma 3.1 and Corollary 3.4, $X$ is transitive on $\Gamma_{1}\left(C\left(A_{q}\right)\right)$ and, as mentioned above, $X$ is also transitive on $\Gamma_{1}(R)$. In particular, $X$ fixes $\Gamma_{1}(C)$ setwise. It follows from Lemma 3.3 that any element of $X$ either fixes $C$ setwise, or sends it to $C^{\prime}=C\left(S_{q} \backslash A_{q}\right) \cup R$. Thus $(C, X)$ is an elusive pair with parameters $(q, q, 3)$. There are, however, two $X$-orbits in $\Gamma_{1}(C)$, so $X$ is not transitive on $\Gamma_{1}(C)$.

Let $G=\operatorname{Aut}(\Gamma)_{\Gamma_{1}(C)}$, the setwise stabiliser in $\operatorname{Aut}(\Gamma)$ of $\Gamma_{1}(C)$. We now show that $X=G$. By [8], since $\delta_{R} \geq 5, \operatorname{Aut}(\Gamma)_{\Gamma_{1}(R)}=\operatorname{Aut}(R)$ and, by [7, Theorem 3.2], $\operatorname{Aut}(R)=X$. Suppose there exists $x \in G \backslash X$. Then because $\Gamma_{1}\left(C\left(A_{q}\right)\right)$ and $\Gamma_{1}(R)$ are both $X$-orbits, it follows that $G$ acts transitively on $\Gamma_{1}(C)$. Therefore, the number, $\left|\Gamma_{1}(\mu) \cap \Gamma_{1}(C)\right|$, of neighbours of the code adjacent to $\mu \in \Gamma_{1}(C)$ is independent of the choice of $\mu$.

Now we inspect the neighbours of $\mu=(1,1,3,4, \ldots) \in \Gamma_{1}(C)$. Changing the first entry to 2 gives us a vertex in $\Gamma_{2}(C)$, but the other $q-2$ choices give us a vertex in $\Gamma_{1}(C)$. Changing the second entry to 2 gives us the codeword $\alpha(1)$, however the other $q-2$ choices give vertices in $\Gamma_{1}(C)$. For $3 \leq i \leq q$, replacing the $i$ th entry with 2 gives us a vertex in $\Gamma_{1}(C)$, while the other $q-2$ choices give vertices in $\Gamma_{2}(C)$. Thus $\left|\Gamma_{1}(\mu) \cap \Gamma_{1}(C)\right|=3(q-2)$. Now let $v=(2,1,1,1, \ldots) \in \Gamma_{1}(C)$. The adjacent vertex with 1 in the first entry is in $C$, but the $q-2$ other vertices that differ in the first entry are in $\Gamma_{1}(C)$. Changing any other entry gives a vertex which is always in $\Gamma_{2}(C)$, since $q \geq 5$. Thus $\left|\Gamma_{1}(v) \cap \Gamma_{1}(C)\right|=q-2 \neq\left|\Gamma_{1}(\mu) \cap \Gamma_{1}(C)\right|$, which is a contradiction.

The first section of the proof of Proposition 3.10 also shows that the image of $C$ under any $x \in X$ that does not fix $C$ is $C^{\prime}=C\left(S_{q} \backslash A_{q}\right) \cup \operatorname{Rep}(q, q)$, and we note that $C \cap C^{\prime}=\operatorname{Rep}(q, q) \neq \emptyset$.

3.4. Nonexistence of elusive codes with parameters $(4,3,3)$. Now we proceed to show that it is not possible to have an elusive code of length four, with minimum 
distance three and an alphabet size three. First we introduce some results and notation from [8].

We say that two codes, $C$ and $C^{\prime}$, in $H(m, q)$, are equivalent if there exists $y \in \operatorname{Aut}(\Gamma)$ such that $C^{y}=C^{\prime}$. Note that this is a more general definition of equivalence than is standard in coding theory, which often requires the number of nonzero entries to be preserved [10, Sections 1.6 and 1.7]. Equivalence preserves minimum distance (see [8, Lemma 4]). The next lemma is an extension of [8, Lemma 1].

Lemma 3.11. If $\alpha$ and $\beta$ are in $H(m, q)$ with $d(\alpha, \beta)=2$, then there are precisely two distinct vertices, $\mu$ and $v$, in $\Gamma_{1}(\alpha) \cap \Gamma_{1}(\beta)$, and $d(\mu, v)=2$. Moreover, given $\alpha$, $\mu$ and $v$ there is only one possible choice for $\beta$.

Proof. By [8, Lemma 1], $\left|\Gamma_{1}(\alpha) \cap \Gamma_{1}(\beta)\right|=2$. We know $\mu, v \in \Gamma_{1}(\alpha) \cap \Gamma_{1}(\beta)$ each differ from $\alpha$ in one entry, say $\mu_{i} \neq \alpha_{i}$ and $v_{j} \neq \alpha_{j}$. If $i \neq j$, then $d(\mu, v)=2$. Suppose $i=j$. Then $\beta_{i}$ is not equal to at least one of $\mu_{i}$ or $v_{i}$, since $\mu_{i} \neq v_{i}$. We know $d(\beta, \mu)=1$, and so if $\beta_{i} \neq \mu_{i}$ then $\beta_{l}=\mu_{l}=\alpha_{l}$ for $l \neq i$, a contradiction since $d(\alpha, \beta)=2$. A similar argument rules out the case $\beta_{i} \neq v_{i}$, and we are left with $d(\mu, v)=2$ and $\beta_{i}=\mu_{i}, \beta_{j}=v_{j}$ and $\beta_{l}=\alpha_{l}$ for $l \neq i, j$.

Let $(C, X)$ be an elusive pair in $H(m, q)$ with $\delta \geq 3$. Suppose $\alpha \in C$ and $x \in X$ such that $\alpha^{x} \notin C$. A pre-codeword of $\alpha$ with respect to $x$ is a vertex $\pi$ such that $d(\alpha, \pi)=2$ and $\pi^{x} \in C$ [8, Definition 3]. We denote the set of all pre-codewords of $\alpha$ with respect to $x$ by $\operatorname{Pre}(\alpha, x)$.

Lemma 3.12. Let $(C, X)$ be an elusive pair in $H(m, q)$ with $\delta \geq 3, \alpha \in C, x \in X$ such that $\alpha^{x} \notin C$, and $\pi \in \operatorname{Pre}(\alpha, x)$. Then the following statements are true.

(i) $\quad\left\{\Gamma_{1}(\alpha) \cap \Gamma_{1}\left(\pi^{\prime}\right) \mid \pi^{\prime} \in \operatorname{Pre}(\alpha, x)\right\}$ forms a partition of $\Gamma_{1}(\alpha)$.

(ii) $\left\{\Gamma_{1}(\pi) \cap \Gamma_{1}(\beta) \mid \beta \in \Gamma_{2}(\pi) \cap C\right\}$ forms a partition of $\Gamma_{1}(\pi)$.

(iii) Let $\mu, v \in \Gamma_{1}(\alpha) \cap \Gamma_{1}(\pi)$ differ from $\alpha$ in entries $i, j$ respectively, and $\pi^{\prime} \in$ $\operatorname{Pre}(\alpha, x) \backslash \pi$. Then there exists $v^{\prime} \in \Gamma_{1}(\alpha) \cap \Gamma_{1}\left(\pi^{\prime}\right)$ that differs from $\alpha$ in some entry $k \neq i, j$.

Proof. For a proof of (i) see [8, Lemma 6(i)], and of (ii) see [8, Lemma 7(ii)].

For part (iii), $d\left(\alpha, \pi^{\prime}\right)=2$, so $\alpha$ and $\pi^{\prime}$ differ in exactly two entries $i^{\prime}$ and $j^{\prime}$. If $\{i, j\}=\left\{i^{\prime}, j^{\prime}\right\}$, then $d\left(\pi, \pi^{\prime}\right)=1$ or 2 , since $\pi \neq \pi^{\prime}$. However $d\left(\pi, \pi^{\prime}\right)=d\left(\pi^{x}, \pi^{\prime x}\right) \geq 3$, since $\pi^{x}, \pi^{\prime x} \in C$ and $\delta \geq 3$. So there must be some value $k \notin\{i, j\}$ and then, by Lemma 3.11, vertex $v^{\prime} \in \Gamma_{1}(\alpha) \cap \Gamma_{1}\left(\pi^{\prime}\right)$ with $v_{l}^{\prime}=\alpha_{l}$, for $l \neq k$, and $v_{k}^{\prime}=\pi_{k}^{\prime}$.

Note that, by [8, Lemma 1], each part in the above partitions has size 2, since the distance between a codeword and pre-codeword is 2 . The partitions themselves have size $m(q-1) / 2$, by [8, Lemma 6(ii)] and [8, Lemma 7(iii)].

Lemma 3.13. There is no elusive pair with parameters $(4,3,3)$.

Proof. Let $(C, X)$ be an elusive pair with parameters $(4,3,3)$. By replacing $C$ with an equivalent code if necessary, we can assume that $\mathbf{0}=0000 \in C$ and that there exists $x \in X$ such that $\mathbf{0}^{x} \notin C$. First we determine, up to equivalence, four members 
of $\operatorname{Pre}(\mathbf{0}, x)$. By [8] it follows that $|\operatorname{Pre}(\mathbf{0}, x)|=4$ and that $\Gamma_{1}(\pi) \subseteq \Gamma_{1}(C)$ for each $\pi \in \operatorname{Pre}(\mathbf{0}, x)$. By Lemma 3.12(i), $\mathcal{P}_{0}=\left\{\Gamma_{1}(\mathbf{0}) \cap \Gamma_{1}(\pi) \mid \pi \in \operatorname{Pre}(\mathbf{0}, x)\right\}$ forms a partition of $\Gamma_{1}(\mathbf{0})$, and by Lemma 3.11, each part of this partition consists of two vertices.

Consider $\left\{1000, v_{1}\right\} \in \mathcal{P}_{0}$. By Lemma 3.11, $v_{1} \neq 2000$. Thus, by replacing $C$ with an equivalent code if necessary, we can assume that $v_{1}=0100$ and, again by Lemma 3.11, $\pi_{1}=1100 \in \operatorname{Pre}(\mathbf{0}, x)$. Next, consider $\left\{2000, v_{2}\right\} \in \mathcal{P}_{0}$. By Lemma 3.12(iii), $v_{2} \neq 0200$, and so again, using the symmetries of the Hamming graph, we can assume that $v_{2}=0020$. Therefore, $\pi_{2}=2020 \in \operatorname{Pre}(\mathbf{0}, x)$. Next, consider $\left\{0200, v_{3}\right\} \in \mathcal{P}_{0}$. If $v_{3}=0010$, this implies that $\{0001,0002\} \in \mathcal{P}_{0}$, contradicting Lemma 3.11. Thus, as before, we can assume that $\nu_{3}=0002$ and $\pi_{3}=0202 \in \operatorname{Pre}(\boldsymbol{0}, x)$. Consequently we deduce that $\pi_{4}=0011 \in \operatorname{Pre}(\mathbf{0}, x)$.

Next we determine three additional elements of $C$. By Lemma 3.12(ii), $\mathcal{P}_{1}=$ $\left\{\Gamma_{1}\left(\pi_{1}\right) \cap \Gamma_{1}(\alpha) \mid \alpha \in \Gamma_{2}\left(\pi_{1}\right) \cap C\right\}$ forms a partition of

$$
\Gamma_{1}\left(\pi_{1}\right)=\left\{\begin{array}{llll}
0100 & 1000 & 1110 & 1101 \\
2100 & 1200 & 1120 & 1102
\end{array}\right\},
$$

and by Lemma 3.11, each part has size 2. We know that $\{1000,0100\} \in \mathcal{P}_{1}$ as $\mathbf{0} \in \Gamma_{2}\left(\pi_{1}\right) \cap C$. Furthermore, by Lemma 3.11, \{1110,1120\} and $\{1101,1102\}$ are not elements of $\mathcal{P}_{1}$. This implies that at least one of 1110 or 1120 forms an element of $\mathcal{P}_{1}$ with either 1101 or 1102 . Thus at least one of $1111,1121,1122$ or 1112 is a codeword in $\Gamma_{1}\left(\pi_{1}\right) \cap C$. Consider $1020 \in \Gamma_{1}\left(\pi_{2}\right)$, which must be adjacent to a codeword with three nonzero entries, as $\delta=3$ and $\mathbf{0} \in C$. Such a codeword has the form $1 a 2 b$, and is at distance at least 2 from 1121 and 1122 , so these vertices are not codewords. By considering $0102 \in \Gamma_{1}\left(\pi_{3}\right)$, a similar argument shows that 1112 is not a codeword either. Thus $1111 \in C$ and $\{1110,1101\} \in \mathcal{P}_{1}$. Consider the part $\{2100, v\} \in \mathcal{P}_{1}$. By Lemma 3.12(iii), $v \neq 1200$, and if $v=1120$ then $2120 \in C$, contradicting the fact that $2120 \in \Gamma_{1}\left(\pi_{2}\right)$. Thus $v=1102$, which leaves $\{1200,1120\} \in$ $\mathcal{P}_{1}$. Hence $\Gamma_{2}\left(\pi_{1}\right) \cap C=\{0000,1111,1220,2101\}$. Finally, consider the partition $\mathcal{P}_{2}=\left\{\Gamma_{1}\left(\pi_{2}\right) \cap \Gamma_{1}(\alpha) \mid \alpha \in \Gamma_{2}\left(\pi_{2}\right) \cap C\right\}$ of

$$
\Gamma_{1}\left(\pi_{2}\right)=\left\{\begin{array}{llll}
0020 & 2120 & 2000 & 2021 \\
1020 & 2220 & 2010 & 2022
\end{array}\right\} .
$$

As $\mathbf{0}, 1220 \in \Gamma_{2}\left(\pi_{2}\right) \cap C$ it follows that $\{0020,2000\},\{1020,2220\} \in \mathcal{P}_{2}$. Consider the part $\{2021, \mu\} \in \mathcal{P}_{2}$. By Lemma 3.11, $\mu \neq 2022$. Thus $\mu=2120$ or 2010 and so $\alpha=2121$ or $2011 \in C$ respectively. However, in both cases $d(\alpha, 1111)=2$, which is a contradiction. Thus no such elusive pair exists.

\section{References}

[1] R. F. Bailey, 'Error-correcting codes from permutation groups', Discrete Math. 309 (2009), 4253-4265.

[2] I. F. Blake, 'Permutation codes for discrete channels', IEEE Trans. Inform. Theory 20 (1974), 138-140. 
[3] I. F. Blake, G. Cohen and M. Deza, 'Coding with permutations', Inf. Control 43 (1979), 1-19.

[4] A. E. Brouwer, A. M. Cohen and A. Neumaier, Distance-Regular Graphs, Ergebnisse der Mathematik und ihrer Grenzgebiete (3) [Results in Mathematics and Related Areas (3)], 18 (Springer, Berlin, 1989).

[5] W. Chu, C. J. Colbourn and P. Dukes, 'Constructions for permutation codes in powerline communications', Des. Codes Cryptogr. 32(1-3) (May 2004), 51-64.

[6] N. I. Gillespie, Neighbour Transitivity on Codes in Hamming Graphs, PhD Thesis, The University of Western Australia, Perth, Australia, 2011.

[7] N. I. Gillespie and C. E. Praeger, 'From neighbour transitive codes to frequency permutation arrays'. 2012. arXiv:1204.2900v1.

[8] N. I. Gillespie and C. E. Praeger, 'Neighbour transitivity on codes in Hamming graphs', Des. Codes Cryptogr., published online February 2012. doi:10.1007/s10623-012-9614-5.

[9] S. Huczynska, 'Powerline communication and the 36 officers problem', Phil. Trans. R. Soc. A 364 (2006), 3199-3214.

[10] W. C. Huffman and V. Pless, Fundamentals of Error Correcting Codes (Cambridge University Press, Cambridge, 2003).

[11] A. J. H. Vinck, 'Coded modulation for power line communications', AEÜ J., 45-49, January 2000.

DANIEL R. HAWTIN, The University of Western Australia,

35 Stirling Highway, Crawley, WA 6009, Australia

e-mail: dan.hawtin@gmail.com

NEIL I. GILLESPIE, The University of Western Australia, 35 Stirling Highway, Crawley, WA 6009, Australia

e-mail: neil.gillespie@uwa.edu.au

CHERYL E. PRAEGER, The University of Western Australia, 35 Stirling Highway, Crawley, WA 6009, Australia

and

Also affiliated with King Abdulaziz University, Jeddah, Saudi Arabia

e-mail: cheryl.praeger@uwa.edu.au 